\title{
THE ASYMPTOTIC BEHAVIOR OF THE SOLUTIONS OF DEGENERATE PARABOLIC EQUATIONS
}

\author{
CATHERINE BANDLE, M. A. POZIO AND ALBERTO TESEI
}

\begin{abstract}
Existence of stationary states is established by means of the method of upper and lower solutions. The structure of the solution set is discussed and a uniqueness property for certain classes is proved by a generalized maximum principle. It is then shown that all solutions of the parabolic equation converge to a stationary state.
\end{abstract}

1. Introduction. Let $D \subset \mathbf{R}^{\mathcal{N}}$ be a bounded, open, connected set with boundary $\partial D \in C^{1}$. Denote by $x$ a generic point and let $w_{0} \in L^{\infty}(D), \chi \in C^{1}(\partial D)$ be two nonnegative functions. This paper deals with degenerate parabolic problems of the following type:

$$
\begin{cases}w_{t}-\Delta \Phi(w)=a(x) f(w) & \text { in } D \times(0, T), \\ w=\chi & \text { on } \partial D \times(0, T), \\ w=w_{0} & \text { in } D \times\{0\},\end{cases}
$$

where

(i) $\Phi \in C^{1}[0, \infty)$ is an increasing function satisfying $\Phi(0)=\Phi^{\prime}(0)=0$, whose inverse $\Phi^{-1}$ is Hölder continuous;

(ii) $f \in C^{1}[0, \infty)$ is also increasing and $f(0)=0$;

(iii) $a \in C^{\alpha}(\bar{D})$ is an arbitrary function changing sign in $\bar{D}, \alpha \in(0,1)$.

We shall be interested only in nonnegative solutions of $(\mathrm{P})$. Problems of this type arise in population dynamics $[\mathbf{N}, \mathbf{G M}]$ and in reaction-diffusion processess. The investigations of this paper were motivated by the special case

$$
w_{t}-\Delta\left(w^{m}\right)=a(x) w^{p} \quad \text { in } D \times(0, T)
$$

where $m>1, p \geq 1$ and $m>p$; this was proposed by Namba [N] as a mathematical model of population dynamics. The existence of an equilibrium solution for $D=$ $\mathbf{R}^{N}$ was proved in $[\mathbf{S c h}]$ and its uniqueness was established in $[\mathbf{S p}]$. Peletier and Tesei $[\mathbf{P e T}]$ used a shooting method to handle the one-dimensional stationary case; Pozio and Tesei [PT] studied problems of this type in a more general context.

We first describe the set of equilibrium solutions for a general class of nonlinearities $\Phi$ and $f$ which includes $\Phi(s)=s^{m}$ and $f(s)=s^{p}$.

An equilibrium solution of problem (P) is an element $w \in L^{\infty}(D)$ which satisfies

$$
-\int_{D} \Phi(w) \Delta \eta d x+\oint_{\partial D} \Phi(\chi) \frac{\partial \eta}{\partial n} d s=\int_{D} a(x) f(w) \eta d x
$$

Received by the editors May 5, 1986 and, in revised form, August 6, 1986.

1980 Mathematics Subject Classification (1985 Revision). Primary 35K60, 35K65; Secondary $35 \mathrm{~J} 65,35 \mathrm{~B} 50$. 
for any $\eta \in C^{2}(\bar{D})$ such that $\eta=0$ on $\partial D$. By an upper solution of the stationary problem (1.2) (namely, by an equilibrium upper solution of $(\mathrm{P})$ ) we mean any $\bar{w} \in L^{\infty}(D)$ which satisfies (1.2) with the inequality sign $\geq$, for any positive $\eta$ as above. Similarly, $\underline{w} \in L^{\infty}(D)$ is called a lower solution of $(1.2)$ if the inequality sign is reversed.

The plan of the first part is to use a modified version of the classical maximum principle to estimate from above the number of the equilibrium solutions. The existence is then established by means of the method of upper and lower solutions. In a further step we embed problem $(\mathrm{P})$ in a family of problems $(\mathrm{P})_{\lambda}$ depending on a positive parameter $\lambda$. It turns out that for small and large values of $\lambda$ a complete picture of the number of stationary solutions can be obtained, whereas for $\lambda$ ranging in some interval $\left(\lambda_{0}, \lambda_{1}\right)$ only estimates are available.

Monotonicity methods are also used to investigate the attractivity properties of equilibrium solutions of $(\mathrm{P})$. Our main result in this respect is that, under rather mild assumptions on $a$, all solutions of $(\mathrm{P})$ converge to a stationary state. A function $w \in C\left([0, T] ; L^{1}(D)\right) \cap L^{\infty}\left(Q_{T}\right)$ is called a solution of $(\mathrm{P})$ if it satisfies (1.3)

$$
\begin{gathered}
\int_{D} w(x, T) \sigma(x, T) d x-\int_{Q_{T}}\left[w \sigma_{t}+\Phi(w) \Delta \sigma\right] d x d t+\int_{0}^{T} d t \oint_{\partial D} \Phi(\chi) \cdot \frac{\partial \sigma}{\partial n} \cdot d s \\
=\int_{D} w_{0} \sigma(x, 0) d x+\int_{Q_{T}} a f(w) \sigma(x, t) x d t
\end{gathered}
$$

for all $\sigma \in C^{2}\left(\bar{Q}_{T}\right)$ with $\sigma=0$ on $\partial D \times(0, T)$. Here we have put $Q_{T}:=D \times$ $(0, T), \partial / \partial n$ stands for the outer normal derivative at $\partial D$. The local existence and uniqueness of solutions of $(\mathrm{P})$ (in the above referred sense) is known (see $[\mathbf{A C P}]$ and the references therein). The investigations concerning the time dependent solutions of $(\mathrm{P})$ rely on the following result $[\mathbf{d M S T}]$.

LEMMA 1.1. Suppose that $\underline{w}_{0}$ and $\bar{w}_{0}$ are lower and upper solutions of the stationary problem $(1.2)$ such that $\underline{w}_{0} \leq \bar{w}_{0}$ a.e in $D$. Let $w\left(x, t ; w_{0}\right)$ stand for the solution of $(\mathrm{P})$.

(i) If $\underline{w}_{0} \leq w_{0} \leq \bar{w}_{0}$ a.e. in $D$, then $w\left(\cdot, t ; w_{0}\right)$ exists for all $t>0$ and satisfies $\underline{w}_{0} \leq w\left(\cdot, t ; w_{0}\right) \leq \bar{w}_{0}$ a.e. in $D$.

(ii) The mapping $t \rightarrow w\left(x, t ; \underline{w}_{0}\right)$ is nondecreasing for a.e. $x \in D$. The mapping $t \rightarrow w\left(x, t ; \bar{w}_{0}\right)$ is nonincreasing for a.e. $x \in D$.

(iii) $w\left(\cdot, t ; \underline{w}_{0}\right)$ converges to a stationary solution $w^{*}$ and $w\left(\cdot, t ; \bar{w}_{0}\right)$ converges to the stationary solution $W^{*}$ (which are the minimal and maximal solutions of (1.2), respectively, in the interval set $\left.K:=\left\{w \in L_{+}^{\infty}(D): \underline{w}_{0} \leq w \leq \bar{w}_{0}\right\}\right)$ as $t \rightarrow \infty$.

The main idea for showing the convergence of all solutions of $(\mathrm{P})$ is to construct upper and lower solutions such that $\underline{w}_{0} \leq w_{0} \leq \bar{w}_{0}$ and to apply the above result. If the equilibrium solution is unique then it attracts all solutions of $(\mathrm{P})$. This is the case when $a(\cdot)$ is of constant sign and $f \circ \Phi^{-1}$ is concave. However, when $a(\cdot)$ changes sign many equilibrium solutions may exist. In fact, we have constructed an example (see §2.3) with infinitely many positive solutions. It turns out that the stationary states can be classified in a natural way and that in each of these classes there is at most one solution. This is an important fact for proving convergence. 


\section{Stationary case.}

2.1. Classification of the solution and uniqueness results. Let $g:=f \circ \Phi^{-1}$ and $\phi:=\Phi[\chi(\cdot)]$. If $w$ is a time independent solution of $(\mathrm{P})$, then $u:=\Phi(w)$ satisfies (in the classical sense)

$$
\Delta u+a(x) g(u)=0 \quad \text { in } D, \quad u=\phi \quad \text { on } \partial D .
$$

It follows immediately from the assumptions of the previous section that $g$ has the following properties:

$$
\begin{aligned}
& g \in C^{\alpha}([0, \infty)) \cap C^{1}((0, \infty)) \quad \text { for some } \alpha \in(0,1) \text { and } \\
& g(s) \geq 0 \text { for } s>0 ; \\
& g^{\prime}(s)>0 \quad \text { for } s>0 ; \\
& g(0)=0 .
\end{aligned}
$$

In addition, we shall assume that

$$
\begin{gathered}
h(s):=\int_{0}^{S}(1 / g(\sigma)) d \sigma \text { exists and } h(0)=0 ; \\
g(s) \quad \text { is strictly concave in }(0, \infty) .
\end{gathered}
$$

In the first part we shall study the question of uniqueness for the solutions of $\left(\mathrm{P}_{s}\right)$. The case $a \geq 0$ has already, been treated by Laetsch $[\mathbf{L}]$, who has shown that there exists at most one nontrivial solution. The same is true when $a \leq 0$. Indeed, if there were two different solutions $u_{1}, u_{2}$, we would have $u_{1}>u_{2}$ in $D^{\prime} \subseteq D, u_{1}=u_{2}$ on $\partial D^{\prime}$. By (A-2) it would then follow that $\Delta\left(u_{1}-u_{2}\right) \geq 0$ in $D^{\prime}$, which implies $u_{1} \leq u_{2}$ in $D^{\prime}$. Obviously this contradicts our assumption.

The situation is more involved when $a(\cdot)$ changes sign. Consider the open sets:

$$
\begin{aligned}
& D^{+}:=\{x \in D: a(x)>0\}, \\
& D^{-}:=\{x \in D: a(x)<0\}
\end{aligned}
$$

and

$$
D^{0}:=\operatorname{int}\{x \in D: a(x)=0\} .
$$

In view of the continuity of $a(\cdot), D^{+}$consists of a countable number of connected components $D_{k}^{+}, k \in \mathbf{M}:=\{1,2, \ldots, r\}$, where $r \leq \infty$.

Throughout this paper it is assumed that

all points $x \in \partial D^{+} \cap D$ satisfy an inner sphere condition with respect to $D^{+}$.

The following observations were already made by Pozio and Tesei $[\mathbf{P T}]$ for a different class of problems.

LEMMA 2.1. Let $u$ be a nonnegative solution of $\left(\mathrm{P}_{s}\right)$.

(i) Either $u \equiv 0$ or $u>0$ in $D_{k}^{+}$.

(ii) If $u>0$ in $D_{k}^{+}$, then $u>0$ in $\bar{D}_{k}^{+} \cap D$.

ProOF. (i) Since $\Delta u \leq 0$ in $D_{k}^{+}, u \geq 0$ on $\partial D_{k}^{+}, u$ cannot attain its minimum $u=0$ in an interior point unless $u=0$ in $D_{k}^{+}$. 
(ii) Let $u\left(x_{0}\right)=0$ for some $x_{0} \in \partial D_{k}^{+} \cap D$. Since $u$ attains there its minimum we must have $\nabla u\left(x_{0}\right)=0$. On the other hand, in view of $(\mathrm{H})$, Hopf's strong maximum principle applies which asserts that $\left|\nabla u\left(x_{0}\right)\right| \neq 0$. This proves (ii).

This lemma gives rise to the following classification of the positive solutions of $\left(\mathrm{P}_{s}\right)$.

DEFINITION 2.1. (i) For any subset $I \subseteq \mathbf{M}$ let $\mathbf{S}_{I}$ be the class of solutions of $\left(\mathrm{P}_{s}\right)$ which are positive in $D_{I}^{+}:=\bigcup_{k \in I} D_{k}^{+}$.

(ii) $\mathbf{N}_{I}$ denotes the set $\left\{u \in \mathbf{S}_{I}: u \equiv 0\right.$ on $\left.D^{+}-D_{I}^{+}\right\}$. We are now in position to formulate the main result of this part.

THEOREM 2.1. Let $g$ satisfy (A-1)-(A-5). Then for any finite $I \subseteq \mathbf{M}, \mathbf{N}_{I}$ has at most one element. If $I \subseteq \mathbf{M}$ is infinite the statement remains true provided that $\bar{D}^{+} \subseteq\left(\bigcup_{k \in \mathbf{M}} \bar{D}_{k}^{+}\right) \cup \partial D$.

The proof of the theorem is based on an elementary lemma already used by Spruck $[\mathbf{S p}]$ in a slightly different context.

LEMMA 2.2. Let $\varepsilon>0$ be a fixed number and $u$ a solution of $\left(\mathrm{P}_{s}\right)$. Then the function $U=h(u+\varepsilon)$ ( $h$ being defined in (A-4)) satisfies

$$
\Delta U=-g^{\prime}(u+\varepsilon)|\nabla U|^{2}-a(x) g(u) / g(u+\varepsilon) .
$$

PROOF. Follows from a straightforward calculation.

PROOF OF THEOREM 2.1. Suppose there exist two different solutions $u_{1}, u_{2} \in$ $\mathbf{N}_{I}$. Let $D^{\prime}=\left\{x \in D: u_{1}(x)>u_{2}(x)\right\}$. Since $u_{1}=u_{2}$ on $\partial D$, we must have $u_{1}=u_{2}$ on $\partial D^{\prime}$. Let $U_{i}=h\left(u_{i}\right)(i=1,2)$. Because of the monotonicity of $h$ we have

$$
U_{1}>U_{2} \quad \text { in } D^{\prime}, \quad U_{1}=U_{2} \quad \text { on } \partial D^{\prime} .
$$

Hence there exists a point $x_{0} \in D^{\prime}$ where the difference $\delta:=U_{1}-U_{2}$ attains its maximum. Let us now distinguish two cases.

(i) Suppose that $U_{2}\left(x_{0}\right)>0$ for some $x_{0} \in D^{\prime}$ where $\delta$ takes its maximum. Denote by $V$ the maximal connected component of the set $D_{1}^{\prime}:=\left\{x \in D^{\prime}: U_{2}(x)>\right.$ $0\}$ containing $x_{0}$. Then $\delta$ belongs to $C^{2}(V)$ and Lemma 2.2 with $\varepsilon=0$ gives in $V$

$$
\Delta \delta=-g^{\prime}\left(u_{1}\right)\left|\nabla U_{1}\right|^{2}+g^{\prime}\left(u_{2}\right)\left|\nabla U_{2}\right|^{2} .
$$

From (A-5) we deduce that

$$
g^{\prime}\left(u_{1}\right)<g^{\prime}\left(u_{2}\right) \quad \text { in } D^{\prime}
$$

which together with (2.2) implies

$$
\Delta \delta+g^{\prime}\left(u_{2}\right)\left(\nabla\left(U_{1}+U_{2}\right), \nabla \delta\right) \geq 0 \quad \text { in } V .
$$

Since $\delta$ assumes its maximum at an interior point of $V$, the maximum principle entails that $\delta \equiv$ constant in $V$. It then follows that

$$
0=\nabla \delta=\frac{\nabla u_{1}}{g\left(u_{1}\right)}-\frac{\nabla u_{2}}{g\left(u_{2}\right)} \quad \text { in } V .
$$

Inserting (2.5) into (2.2) we get

$$
0=\Delta \delta=\left(\frac{\left|\nabla u_{1}\right|}{g\left(u_{1}\right)}\right)^{2}\left(g^{\prime}\left(u_{2}\right)-g^{\prime}\left(u_{1}\right)\right) \quad \text { in } V .
$$


From (2.3), (2.5) and (2.6) we then conclude that $\nabla u_{1}=\nabla u_{2}=0$ in $V$ and $u_{1} \equiv u_{1}^{*}, u_{2} \equiv u_{2}^{*}$ in $V$, where $u_{1}^{*}>u_{2}^{*}$ are two positive constants. On the boundary of $V$ we have either $U_{2}=0$ or $U_{2}=U_{1}$. This implies that either $u_{2}=u_{2}^{*}=0$ or $u_{2}^{*}=u_{1}^{*}$. Both cases lead to a contradiction.

(ii) Suppose now $U_{2}\left(x_{0}\right)=0$ for all $x_{0}$ where $\delta$ achieves its maximum. Let

$$
C:=\left\{x \in D^{\prime}: \delta(x)=\delta\left(x_{0}\right)\right\} .
$$

Note that by assumption $U_{2} \equiv 0$ in $C$; since

$$
\delta=\delta\left(x_{0}\right)>0 \quad \text { in } C,
$$

we have $U_{1}>0$ in $C$. On the other hand, $u_{1} \in \mathbf{N}_{I}$ implies $u_{1} \equiv 0$ in $\overline{D^{+}-D_{I}^{+}}$. Hence by (2.7) and the monotonicity of $h$ we get

$$
C \cap \overline{\left(D^{+}-D_{I}^{+}\right)}=\varnothing \text {. }
$$

According to Lemma 2.1(ii) we also have $U_{2}>0$ in $\bar{D}_{k}^{+} \cap D \forall k \in I$. If $\partial D_{k}^{+} \cap \partial D$ is nonempty for some $k \in I$, we have $u_{1}=u_{2}$, thus $\delta \equiv 0$ in such intersection. Hence

$$
C \cap \bar{D}_{k}^{+}=\varnothing \quad \text { for all } k \in I .
$$

(2.8) and (2.9) imply that (since $I$ is finite by hypothesis)

$$
C \cap \bar{D}^{+}=\varnothing \text {. }
$$

$C$ and $\bar{D}^{+}$are therefore at a positive distance from each other. Then there exists a connected neighborhood $U \supset C$ such that $\bar{U} \cap \bar{D}^{+}=\varnothing$ and $\delta(x)>0$ in $\bar{U}$. The monotonicity of $h$ implies that

$$
\min _{\bar{U}}\left(u_{1}(x)-u_{2}(x)\right)>0
$$

where $W$ is a connected component of $U$.

Thus there exists $b>0$ such that $\delta(x) \leq b<\delta\left(x_{0}\right)$ for any $x \in \partial W$. For any $\varepsilon>0$ define

$$
U_{2 \varepsilon}:=h\left(u_{2}+\varepsilon\right), \quad \delta_{\varepsilon}:=U_{1}-U_{2 \varepsilon} .
$$

Clearly $\delta_{\varepsilon} \leq \delta$ in $D$. By the previous remarks there exists $\varepsilon>0$ such that

$$
u_{1}>u_{2}+\varepsilon \quad \text { in } \bar{W} \quad \text { and } \quad \delta_{\varepsilon}\left(x_{0}\right)>b .
$$

It then follows that $\delta_{\varepsilon}(x) \leq \delta(x) \leq b<\delta_{\varepsilon}\left(x_{0}\right)$ for any $x \in \partial W$. Hence $\delta_{\varepsilon}$ attains its maximum at some interior point in $W$ and is not constant in $\bar{W}$. On the other hand, Lemma 2.2 implies, in view of (2.11) and (A-5)

$$
\Delta \delta_{\varepsilon} \geq-g^{\prime}\left(u_{2}+\varepsilon\right)\left(\nabla\left(U_{1}+U_{2 \varepsilon}\right), \nabla \delta_{\varepsilon}\right)-a(x)\left(1-\frac{g\left(u_{2}\right)}{g\left(u_{2}+\varepsilon\right)}\right) \quad \text { in } W .
$$

Since $\bar{W} \cap \bar{D}^{+}$is empty, we have $a \leq 0$ in $\bar{W}$, and therefore

$$
\Delta \delta_{\varepsilon}+g^{\prime}\left(u_{2}+\varepsilon\right)\left(\nabla\left(U_{1}+U_{2 \varepsilon}\right), \nabla \delta_{\varepsilon}\right) \geq 0 \quad \text { in } W .
$$

By the maximum principle $\delta_{\varepsilon}$ cannot achieve its maximum in $W$ unless it is constant. This is a contradiction, whence the result follows. 
2.2. Existence of solutions in $\mathbf{S}_{I}$. The results of this section hold under the following conditions on $g$ :

$$
\lim _{s \rightarrow \infty} g(s) / s=0 \quad \text { (sublinearity at infinity); }
$$

$$
g(0)=0 \quad \text { and } \lim _{s \rightarrow 0^{+}} g(s) / s=\infty \quad \text { (superlinearity at zero). }
$$

Observe that (A-3) and (A-4) imply (A-7).

The next lemma has already been derived in [PT]. Since it is important for our investigation, we shall repeat its proof.

LEMMA 2.3. Assume (A-6) and (A-7). in $\bar{D}$.

(i) For any $M>0$ there exists a stationary upper solution $\bar{u}$ such that $\bar{u} \geq M$

(ii) For any $I \subseteq \mathbf{M}$ and any open set $U$ such that $\bar{U} \subseteq D_{I}^{+}, \bar{U} \cap D_{k}^{+} \neq \varnothing \forall k \in I$, there exists a family of lower solutions $\underline{u}_{\rho}, \rho \in\left(0, \rho_{0}\right]$, such that $\operatorname{supp} \underline{u}_{\rho} \subseteq U$, $\operatorname{supp} \underline{u}_{\rho} \cap D_{k}^{+} \neq \varnothing \forall k \in I$ and $\lim _{\rho \rightarrow 0}\left\|\underline{u}_{\rho}\right\|_{\infty}=0$.

ProOF. (i) Let $B$ be an open ball, $B \supset \bar{D}$ and $\xi>0$ be the first eigenfunction of

$$
\Delta \xi+\lambda \xi=0 \quad \text { in } B, \quad \xi=0 \quad \text { on } \partial B .
$$

Then $\xi \geq \varepsilon>0$ on $\partial D$, thus by construction we have $\xi \geq \varepsilon$ in $\bar{D}$. Since

$$
\Delta(\nu \xi)+a(x) g(\nu \xi)=\left[-\lambda+a(x) \frac{g(\nu \xi)}{\nu \xi}\right] \nu \xi
$$

there exists by (A-6) a number $\nu_{0}>0$ such that $\Delta(\nu \xi)+a(x) g(\nu \xi) \leq 0$ in $D, \nu \xi \geq \phi$ on $\partial D$ for all $\nu \geq \nu_{0}$. Hence $\nu \xi=: \bar{u}$ is an upper solution and for $\nu$ sufficiently large we have $\bar{u}>M$ in $D$. This proves assertion (i).

(ii) For $k \in I$ let $\bar{B}_{k}$ be a ball such that $\bar{B}_{k} \subset U \cap D_{k}^{+}$and set $\underline{a}_{k}:=\inf _{B_{k}} a(x)$. Consider the function

$$
\underline{u}_{\rho}= \begin{cases}\rho \xi_{k} & \text { in } B_{k}(\rho>0) \forall k \in I, \\ 0 & \text { elsewhere, }\end{cases}
$$

where $\xi_{k}>0$ is the first eigenfunction of $\Delta \xi_{k}+\lambda_{k} a_{k} \xi_{k}=0$ in $B_{k}, \xi_{k}=0$ on $\partial B_{k}$. Then

$$
\begin{aligned}
&-\int_{D} \underline{u}_{\rho} \Delta \eta d x+\oint_{\partial D} \phi \frac{\partial \eta}{\partial n} d s-\int_{D} a(x) g\left(\underline{u}_{\rho}\right) \eta d x \\
&=\sum_{k \in I}\left\{\int_{B_{k}}\left[\lambda_{k} \underline{a}_{k}-\frac{a g\left(\underline{u}_{\rho}\right)}{\underline{u}_{\rho}}\right] \underline{u}_{\rho} \eta d x+\oint_{\partial B_{k}} \eta \frac{\partial \underline{u}_{\rho}}{\partial n} d s\right\}+\oint_{\partial D} \phi \frac{\partial \eta}{\partial n} d s \\
& \forall \eta \in C^{2}(\bar{D}), \eta \geq 0 \text { in } D, \eta=0 \text { on } \partial D .
\end{aligned}
$$

Since $\partial \underline{u}_{\rho} / \partial n<0$ on $\partial B_{k}$ and $\partial \eta / \partial n \leq 0$ on $\partial D$ for $\eta \geq 0$, it follows for sufficiently small $\rho$, that

$$
\int_{D}\left(\underline{u}_{\rho} \Delta \eta+a g\left(\underline{u}_{\rho}\right) \eta\right) d x-\oint_{\partial D} \phi \frac{\partial \eta}{\partial n} d s \geq 0 \quad \forall \eta \in C^{2}(\bar{D}), \eta \geq 0 \text { in } D, \eta=0 \text { on } \partial D \text {. }
$$

Hence $\underline{u}_{\rho}$ is a lower solution with the desired properties.

The next result is an immediate consequence of the previous lemma [PT, ACP, dMST] and the method of upper and lower solutions. 
THEOREM 2.2. Under assumptions (A-1), (A-6) and (A-7) we have for any $I \subseteq \mathbf{M} \mid$ :

(i) $\mathbf{S}_{I} \neq \varnothing$;

(ii) there exists a minimal and a maximal solution $u_{I}$ and $U_{I}$ in $\mathbf{S}_{I}$, such that $u_{I} \leq u \leq U_{I}$ for any other solution $u \in S_{I}$;

(iii) the maximal solution $U_{I}$ coincides with the maximal element of $\mathbf{S}_{\mathbf{M}}$;

(iv) if the minimal solution differs from $U_{I}$, then $u_{I} \in \mathbf{N}_{\tilde{I}}$, where $\tilde{I}=\bigcap\left\{I^{\prime}: I^{\prime} \supseteq\right.$ $\left.I, \mathbf{N}_{I^{\prime}} \neq \varnothing\right\}$.

ProOF. (i) is a consequence of Lemma 2.3 and the classical method of lower and upper solutions.

(ii) A proof of this assertion can be found in [PT, Theorem 4].

(iii) Suppose that $U_{I} \not \equiv U_{\mathbf{M}}$. Then $U_{I} \equiv 0$ in $D_{k}^{+}$for some $k \in \mathbf{M}-I$. Since $U_{\mathbf{M}}$ belongs to $\mathbf{S}_{I}$, it follows from the maximality of $U_{I}$ that $U_{\mathbf{M}} \leq U_{I}$ and thus $U_{\mathbf{M}} \equiv 0$ in $D_{k}^{+}$contradicting the definition of $U_{\mathbf{M}}$.

(iv) We have $u_{I} \leq u^{\prime}$ for $u^{\prime} \in \mathbf{N}_{I^{\prime}}$ defined above. Hence $u_{I} \equiv 0$ in $D_{\mathbf{M}}^{+}-D_{I^{\prime}}^{+}$ and therefore $u_{I} \in \mathbf{N}_{\tilde{I}}$. Q.E.D.

The maximal solution $U:=U_{\mathbf{M}}$ can be constructed by means of a variational principle. Instead of $\left(\mathrm{P}_{s}\right)$ we consider the problem

$$
\Delta \nu+a(x) g(\nu+h)=0 \quad \text { in } D, \quad \nu=0 \quad \text { on } \partial D
$$

where $\Delta h=0$ in $D, h=\phi$ on $\partial D$. Clearly the function $u=\nu+h$ is a solution of $\left(\mathrm{P}_{s}\right)$. Let

$$
J[w]:=\int_{D}|\nabla w|^{2} d x-2 \int_{D} a(x) G(w+h) d x, \quad G(s):=\int_{0}^{s} g(\tau) d \tau .
$$

THEOREM 2.3. Under the same assumptions as for Theorem 2.2, the following assertions hold:

(i) the problem

$$
J[w]=\inf , \quad w \in H_{0}^{1}(D)
$$

possesses a solution $w_{0}$;

(ii) if in addition $r<+\infty$ and (A-2)-(A-5) hold, then (2.14) is uniquely solvable and $w_{0}+h=U$, where $U$ is the maximal solution of $\left(\mathrm{P}_{s}\right)$.

PROOF. The proof of (i) is standard. In a first step we show that $J[w]$ is bounded from below. Let

$$
\bar{a}:=\left\{\begin{array}{ll}
\max _{\bar{D}} a(x) & \text { if } a(x)>0 \text { for some } x \in D, \\
0 & \text { if } a \leq 0,
\end{array} \quad \bar{h}:=\max _{\bar{D}} h(x) .\right.
$$

Then for any $w \in H_{0}^{1}(D)$

$$
J[w] \geq \int_{D}|\nabla w|^{2} d x-2 \bar{a} \int_{D} G(w+\bar{h}) d x .
$$

By H. Poincaré's inequality

$$
\int_{D}|\nabla w|^{2} d x \geq c_{0} \int_{D} w^{2} d x \quad \text { for all } w \in H_{0}^{1}(D)\left(c_{0}>0\right)
$$


and by (A-6) there exist $s_{0}$ and $c_{1}$ such that $G(s+\bar{h}) \leq\left(c_{0} / 4 \bar{a}\right) \cdot s^{2}+c_{1}$ for all $s \geq s_{0}$. Hence

$$
J[w] \geq \frac{c_{0}}{2} \int_{D} w^{2} d x-2 \bar{a} \int_{D}\left\{G\left(s_{0}+\bar{h}\right)+c_{1}\right\} d x \geq c_{2},
$$

where $c_{2}$ is independent of $w$. Thus there exists a minimizing sequence

$$
\left\{w_{n}\right\}_{n=1}^{\infty} \subset H_{0}^{1}(D) \quad \text { such that } \lim _{n \rightarrow \infty} J\left[w_{n}\right]=\inf _{w \in H_{0}^{1}(D)} J[w]=: d .
$$

This means that for every $\varepsilon>0$ there exists $n_{0}(\varepsilon)$ such that $d+\varepsilon \geq J\left[w_{n}\right] \forall n \geq$ $n_{0}(\varepsilon)$, whence by $(2.15)$

$$
\int_{D}\left|\nabla w_{n}\right|^{2} d x \leq c_{4} \quad \forall n \geq n_{0}(\varepsilon)
$$

We can therefore extract a subsequence $\left\{w_{n_{k}}\right\}_{k=1}^{\infty}$ such that $w_{n_{k}} \rightarrow w_{0}$ weakly in $H_{0}^{1}(D)$ and $w_{n_{k}} \rightarrow w_{0}$ strongly in $L^{2}(D)$. Then standard arguments and Lebesgue's dominated convergence theorem yield

$$
\lim _{k \rightarrow \infty} J\left[w_{n_{k}}\right]=J\left[w_{0}\right], \quad w_{0} \in H_{0}^{1}(D),
$$

which establishes (i). On the other hand, if $w_{0}$ is any solution to (2.14), it follows from the standard regularity arguments that $w_{0}$ solves (2.13). Thus $U:=w_{0}+h$ is a solution of $\left(\mathrm{P}_{s}\right)$. The uniqueness of $w_{0}$ follows as soon as we prove that $U$ is the (unique) maximal solution of $\left(\mathrm{P}_{s}\right)$. Under the additional hypotheses in (ii), by Theorem 2.1, this is equivalent to prove that $U>0$ in $D^{+}$. By contradiction suppose that $U \equiv 0$ in $D_{k}^{+}$for some $k \in \mathbf{M}$. Consider a ball $B \subset D_{k}^{+}$and let $\xi>0$ be the first eigenfunction of $\Delta \xi+\lambda \xi=0$ in $B, \xi=0$ on $\partial B$. Define

$$
\tilde{w}:= \begin{cases}w_{0} & \text { in } D-B \\ -h+\varepsilon \xi & \text { in } B .\end{cases}
$$

Then

$$
J[\tilde{w}]=J\left[w_{0}\right]+\varepsilon^{2} \int_{B}|\nabla \xi|^{2} d x-2 \int_{B} a(x) G(\varepsilon \xi) d x .
$$

Let $a(x) \geq \underline{a}>0$ for any $x$ in $B$ and choose $\varepsilon$ so small that

$$
g(\varepsilon \xi)>(2 \lambda / \underline{a}) \varepsilon \xi \quad \text { in } B .
$$

This is possible in view of $(\mathrm{A}-7)$. Thus $G(\varepsilon \xi) \geq(\lambda / \underline{a}) \varepsilon^{2} \xi^{2}$. Hence

$$
J[\tilde{w}] \leq J\left[w_{0}\right]+\varepsilon^{2} \lambda \int_{B} \xi^{2} d x-\frac{2 \lambda}{\underline{a}} \cdot \varepsilon^{2} \int_{B} a(x) \xi^{2} d x<J\left[w_{0}\right]
$$

contradicting statement (i). Thus (ii) follows.

2.3. On the number of stationary solutions. Let $\lambda \in \mathbf{R}^{+}$and put $a_{\lambda}(x):=$ $a^{+}(x)-\lambda a^{-}(x)$, where $a^{ \pm}(x):=\max \{0, \pm a(x)\}$ and $a^{+} \not \equiv 0$. The aim of this section is to study the number of solutions of

$$
\Delta u+a_{\lambda}(x) g(u)=0 \quad \text { in } D, \quad u=\phi \quad \text { on } \partial D
$$

for various $\lambda>0$. We start with some general remarks about the solutions of $\left(\mathrm{P}_{s}\right)_{\lambda}$. 
LEMMA 2.4. Assume (A-6) and (A-7). Denote by $U_{\lambda}$ and $u_{\lambda}$ the maximal and the minimal solution of $\left(\mathrm{P}_{s}\right)_{\lambda}$ in $\mathbf{S}_{I}, I \subseteq \mathbf{M}$ fixed. Then for any $0<\lambda^{\prime} \leq \lambda$ we have

(i) $U_{\lambda^{\prime}} \geq U_{\lambda}$;

(ii) $u_{\lambda^{\prime}} \geq u_{\lambda}$.

PROOF. (i) Since $a_{\lambda^{\prime}}(x) \geq a_{\lambda}(x)$, it follows that $\Delta U_{\lambda}+a_{\lambda^{\prime}}(x) g\left(U_{\lambda}\right) \geq \Delta U_{\lambda}+$ $a_{\lambda}(x) g\left(U_{\lambda}\right)=0$. Moreover by Lemma 2.3(i) there exists an upper solution $\bar{u} \geq U_{\lambda}$ for $\left(\mathrm{P}_{s}\right)_{\lambda^{\prime}}$. Hence there exists a solution $\bar{u} \geq u \geq U_{\lambda}$ of $\left(\mathrm{P}_{s}\right)_{\lambda^{\prime}}$ and consequently $U_{\lambda^{\prime}} \geq u \geq U_{\lambda}$.

(ii) We have $\Delta u_{\lambda^{\prime}}+a_{\lambda}(x) g\left(u_{\lambda^{\prime}}\right) \leq \Delta u_{\lambda^{\prime}}+a_{\lambda^{\prime}}(x) g\left(u_{\lambda^{\prime}}\right)=0$ and by Lemma 2.3(ii) there exists a lower solution $\underline{u}_{\rho} \leq u_{\lambda}$; hence there is a solution $\underline{u}_{\rho} \leq u \leq u_{\lambda^{\prime}}$ of $\left(\mathrm{P}_{s}\right)_{\lambda}$. Thus $u_{\lambda} \leq u \leq u_{\lambda^{\prime}}$. Similarly we obtain for $\mathbf{N}_{I}(\lambda)$ given in Definition 2.1 with $\left(\mathrm{P}_{s}\right)$ replaced by $\left(\mathrm{P}_{s}\right)_{\lambda}$.

COROllaRY 2.1. If $\mathbf{N}_{I}\left(\lambda_{0}\right) \neq \varnothing$ for some $\lambda_{0}>0$, then $\mathbf{N}_{I}(\lambda) \neq \varnothing$ for all $\lambda \geq \lambda_{0}$.

COROLLARY 2.2. (i) There exists a constant $C$ independent of $\lambda$ such that $u \leq C$ for any solution $u$ of $\left(\mathrm{P}_{s}\right)_{\lambda}$.

(ii) For all $\lambda \leq \lambda_{0}$ and for all solutions $u$ of $\left(\mathrm{P}_{s}\right)_{\lambda}$ in $\mathrm{S}_{I}$ we have $u \geq u_{\lambda_{0}}$, where $u_{\lambda_{0}}$ is the minimal solution of $\left(\mathrm{P}_{s}\right)_{\lambda_{0}}$ in $\mathbf{S}_{I}$.

Proof. (i) From Lemma 2.4(i) we get $u \leq U_{\lambda} \leq U_{0}$, where $U_{0}$ is the maximal solution of $\left(\mathrm{P}_{s}\right)_{0}$. In view of our assumption $a^{+} \not \equiv 0, U_{0} \not \equiv 0$.

(ii) follows from Lemma 2.4(ii).

THEOREM 2.4. Assume (A-1)-(A-6) and $r<\infty$. Then there exists a number $\lambda_{0}>0$ such that $\left(\mathrm{P}_{s}\right)_{\lambda}$ possesses a unique nortrivial solution for all $\lambda \in\left[0, \lambda_{0}\right]$, which moreover is positive in $D^{+}$.

PROOF. As we alreadi know from Theorems 2.2 and 2.1, for each $\lambda \geq 0$ there is a unique solution $U_{\lambda} \in \mathbf{S}_{\mathbf{M}}$. Observe that if $\left(\mathrm{P}_{s}\right)_{\lambda}$ possesses another nontrivial solution $u_{\lambda}$, this one must vanish identically in some $D_{k}^{+}$. Suppose that the assertion of Theorem 2.4 is false. Then there exists a sequence $\left\{\lambda_{n}\right\}_{n=1}^{\infty}, \lambda_{n} \rightarrow 0$, for $n \rightarrow \infty$ such that $u_{\lambda_{n}} \not \equiv U_{\lambda_{n}}$ and hence $u_{\lambda_{n}} \equiv 0$ in $D_{k_{n}}^{+}$. By Corollary 2.2(i) $\left\{u_{\lambda_{n}}\right\}_{n=1}^{\infty}$ is uniformly bounded. If $G(x, y)$ stands for the Green's function of the Laplace operator, which vanishes on the boundary, we have for $u_{\lambda_{n}}$ the integral representation

$$
u_{\lambda_{n}}(x)=\int_{D} G(x, y) a_{\lambda_{n}}(y) g\left(u_{\lambda_{n}}(y)\right) d y-\oint_{\partial D} \frac{\partial G}{\partial n_{y}}(x, y) \phi(y) d s \quad(x \in D) .
$$

Therefore by Corollary 2.2(i)

$$
\begin{aligned}
\left|u_{\lambda_{n}}(x)-u_{\lambda_{n}}(z)\right| \leq & c_{0} \int_{D}|G(x, y)-G(z, y)| d y \\
& +\left|\oint_{\partial D}\left[\frac{\partial G}{\partial n_{y}}(x, y)-\frac{\partial G}{\partial n_{y}}(z, y)\right] \phi(y) d s\right|
\end{aligned}
$$

where $c_{0}$ is independent of $\lambda$. The right-hand side of this inequality tends to zero as $x \rightarrow z$, which proves that $\left\{u_{\lambda_{n}}\right\}_{n=1}^{\infty}$ is equicontinuous. The lemma of 
Arzelà-Ascoli now applies and we can extract a subsequence, say $\left\{u_{\lambda_{n}}\right\}$, which converges uniformly to a continuous function $u_{0}$. Since $\mathbf{M}$ is finite we can choose this subsequence such that $u_{\lambda_{n}} \equiv 0$ in $D_{k_{0}}^{+}$for some fixed $k_{0} \in \mathbf{M}$. Hence $u_{0} \equiv 0$ in $D_{k_{0}}^{+}$. We next observe that

$$
u_{0}(x)=\int_{D} G(x, y) a^{+}(y) g\left(u_{0}\right) d y-\oint_{\partial D} \frac{\partial G}{\partial n_{y}}(x, y) \phi(y) d s \quad(x \in D),
$$

which implies that $u_{0}$ is a solution of $\left(\mathrm{P}_{s}\right)_{0}$. Since $\left(\mathrm{P}_{s}\right)_{0}$ has only one nontrivial solution $U_{0}$, which is positive in $D^{+}$, we must have

$$
u_{0} \equiv 0 \text {. }
$$

On the other hand, $u_{\lambda_{n}}$ was supposed to be a nontrivial solution of $\left(\mathrm{P}_{s}\right)_{\lambda_{n}}$, hence $u_{\lambda_{n}}>0$ in some $D_{j_{n}}^{+}$. Again the finiteness of $\mathbf{M}$ implies that we can choose a subsequence, say $\left\{u_{\lambda_{n}}\right\}$, such that $u_{\lambda_{n}} \rightarrow u_{0}$ and $u_{\lambda_{n}}>0$ in $D_{j_{0}}^{+}$for some $j_{0} \in \mathbf{M}$. From Corollary 2.2(ii) we deduce that $u_{\lambda_{n}} \geq u_{\varepsilon}$ for all $\lambda_{n} \leq \varepsilon$, where $u_{\varepsilon}$ is the minimal solution of $\left(\mathrm{P}_{s}\right)_{\varepsilon}$ in $\mathbf{S}_{\left\{j_{0}\right\}}$. Thus $u_{0} \geq u_{\varepsilon}>0$ in $D_{j_{0}}^{+}$, contradicting (2.16). This establishes the theorem.

REMARK. The next example shows that in general Theorem 2.4 does not hold if $\mathbf{M}$ is infinite.

EXAMPLE. Take $D:=\left\{x \in R^{2}: x_{1}^{2}+x_{2}^{2}<4 \pi^{2}\right\}$ and consider the sequences $\left\{c_{n}\right\}_{n=1}^{\infty}$, defined by $c_{1}:=\pi, c_{n}:=\pi \sum_{k=0}^{n-1} 2^{-k}$, and $\left\{h_{n}\right\}_{n=1}^{\infty}$, where $h_{n}:=1 / 2^{n+1}$. From these definitions it follows that $\lim _{n \rightarrow \infty} c_{n}=2 \pi, \lim _{n \rightarrow \infty} h_{n}=0$ and $c_{n+1}=$ $c_{n}+2 \pi h_{n}$. Let $r=|x|$ and define

$$
\begin{gathered}
a^{+}(r):=\left\{\begin{array}{c}
4 h_{n}^{2}\left[1-4 \cos ^{2}\left(\left(r-c_{n}\right) / h_{n}\right)-h_{n} \sin \left(\left(r-c_{n}\right) / h_{n}\right) \cos \left(\left(r-c_{n}\right) / h_{n}\right) / r\right]^{+} \\
\text {if } c_{n} \leq r \leq c_{n}+\pi h_{n}, n=1,2, \ldots,
\end{array}\right. \\
a^{-}(r):=\left\{\begin{array}{rr}
\text { if } 0 \leq r \leq \pi \text { or } c_{n}+\pi h_{n} \leq r \leq c_{n+1}, n=1,2, \ldots, \\
\text { or } r=2 \pi,
\end{array}\right. \\
\begin{array}{rr}
4 h_{n}\left[1-4 \cos ^{2}\left(\left(r-c_{n}\right) / h_{n}\right)-h_{n} \sin \left(\left(r-c_{n}\right) / h_{n}\right) \cos \left(\left(r-c_{n}\right) / h_{n}\right) / r\right]^{-} \\
3 \quad & \text { if } 0 \leq r \leq \pi, \\
12 h_{n+1}-12\left(h_{n+1}-h_{n}\right)\left(c_{n+1}-r\right) /\left(c_{n+1}-c_{n}-\pi h_{n}\right)
\end{array} \\
\end{gathered}
$$

Consider the problem

$$
\Delta u+a_{\lambda}(x) u^{1 / 2}=0 \quad \text { in } D, \quad u=0 \quad \text { on } \partial D .
$$

Define

$$
\bar{u}(r)=\left\{\begin{array}{c}
h_{n}^{8} \sin ^{4}\left(\left(r-c_{n}\right) / h_{n}\right) \quad \text { if } c_{n} \leq r \leq c_{n}+\pi h_{n}, n=1,2, \ldots \\
0 \quad \text { if } 0 \leq r \leq \pi \text { and } c_{n}+\pi h_{n} \leq r \leq c_{n+1}, n=1,2, \ldots \\
\quad \text { or } r=2 \pi
\end{array}\right.
$$

and

$$
\bar{u}_{n}(r)= \begin{cases}\bar{u}(r) & \text { if } c_{n} \leq r \leq 2 \pi \\ 0 & \text { if } 0 \leq r \leq c_{n}\end{cases}
$$


A straightforward calculation yields

$$
\Delta \bar{u}_{n}+a_{\lambda} \bar{u}_{n}^{1 / 2}= \begin{cases}0 & \text { if } \bar{u}_{n}=0 \text { or } a=a^{+}>0, \\ \left(\lambda-h_{n}\right) a \bar{u}_{n}^{1 / 2} & \text { if } a=-a^{-}<0,\end{cases}
$$

which shows that for $n$ sufficiently large, $\bar{u}_{n}$ is an upper solution. By Lemma 2.3(ii) we can construct a lower solution $\underline{u} \leq \bar{u}_{n}$ such that $\underline{u} \not \equiv 0$ in each connected component of $D^{+} \cap \operatorname{supp} \bar{u}_{n}$ and $\underline{u} \equiv 0$ for $r \leq c_{n}$. Hence for any $\lambda$ there exists a nontrivial solution $\underline{u} \leq u \leq \bar{u}$, whose support has infinitely many connected components. This proves that problem $(2.17)_{\lambda}$ has for any $\lambda$ infinitely many nontrivial solutions whereas $(2.17)_{0}$ has a unique nontrivial solution.

As we have shown in Theorem 2.4, if $r<+\infty \mathrm{N}_{I}(\lambda)$ is empty for small $\lambda$ and for any $I \subset \mathbf{M}$. It seems reasonable to expect that for large $\lambda, \mathbf{N}_{I}(\lambda)$ contains an element. However, this is not always the case, as the following example shows.

Consider the situation in Figure 1.

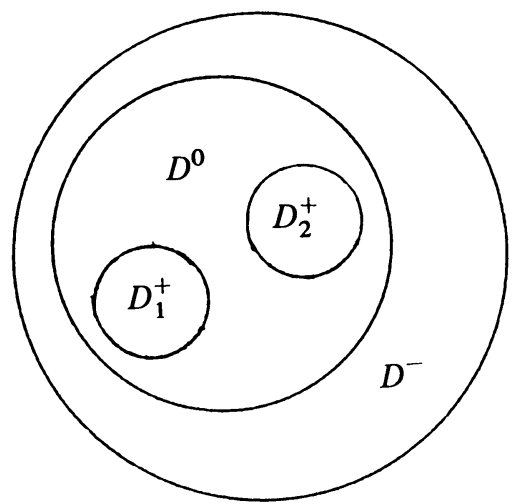

FIGURE 1

Here $\mathbf{N}_{\{1\}}(\lambda)=\mathbf{N}_{\{2\}}(\lambda)=\varnothing$, for any $\lambda \geq 0$. In fact, if $u \in \mathbf{N}_{\{1\}}(\lambda)$, by Lemma 2.1(ii) we have $u>0$ on $\partial D_{1}^{+}$and (by continuity) $u>0$ at some points in $D^{0}$. Due to the regularity of the boundary of $D^{0}$ we assume in this example, the arguments of Lemma 2.1 apply to $D^{0}$, too. Hence $u>0$ in $\overline{D^{0}}$. In particular, $u>0$ on $\partial D_{2}^{+}$, which implies $u \not \equiv 0$ in $D_{2}^{+}$. This contradicts the assumption $u \in \mathbf{N}_{\{1\}}(\lambda)$.

Another case where $\mathbf{N}_{I}(\lambda)=\varnothing$ for all $\lambda$, is when $\phi \not \equiv 0, \partial D \subseteq \partial D_{j}^{+}$and $j \notin I$. In order to prevent such a situation we shall introduce the notion of admissible $I$. More precisely, let $\Gamma^{+}:=\{x \in \partial D: \phi(x)>0\}$; then

DEFINITION 2.2. I $\subset \mathbf{M}$ is called admissible if there exist two closed sets $C_{1}, C_{2} \subseteq \bar{D}$, such that

(i) $C_{1} \cap C_{2}=\varnothing, D_{I}^{+} \cup \Gamma^{+} \subseteq C_{1}, D_{\mathbf{M}-I}^{+} \subseteq C_{2}$;

(ii) $\{x \in \bar{D}: a(x)=0\} \subseteq C_{1} \cup C_{2}$.

THEOREM 2.5. Assume (A-1)-(A-3), (A-5), (A-6) and instead of (A-4) the stronger assumption ${ }^{1}$

$$
\int_{0}^{1} d s / \sqrt{G(s)}<\infty
$$

${ }^{1}$ By $\left(\right.$ A-2) we have $s g(s) \geq G(s)$ and by $(\mathrm{A}-4)^{\prime} \infty=\lim _{s \rightarrow 0^{+}} G(s) / s^{2}=\lim _{s \rightarrow 0^{+}} g(s) / 2 s$. Hence $1 / g(s) \leq 1 / \sqrt{G(s)}$ for $s \leq s_{0}$. 
If $I \subset \mathbf{M}$ is admissible, then there exists $\lambda_{0}>0$ such that $\mathbf{N}_{I}(\lambda) \neq \varnothing$ for all $\lambda \geq \lambda_{0}$.

When proving Theorem 2.5, we shall make use of the following result which appears in [DH, Sch, PT].

LEMMA 2.5. Let $B=\left\{x \in \mathbf{R}^{N}:|x|<\delta\right\}$ and $g>0$ be an increasing function in $[0, \infty)$ satisfying $\int_{0}^{\gamma} d s / \sqrt{G(s)}<\infty$, where $G(s):=\int_{0}^{s} g(\sigma) d \sigma$. Denote by $\bar{u}$ the unique solution of

$$
\Delta \bar{u}=\bar{c}^{2} g(\bar{u}) \quad \text { in } B, \quad \bar{u}=\gamma \quad \text { on } \partial B .
$$

Then there exists a number $c_{0}>0$ such that $\bar{u}(0)=0$ for all $c \geq c_{0}$.

PROOF OF LEMMA 2.5. Consider the ordinary differential equation

$$
z^{\prime \prime}=c^{2} g(z) \text { in }(0, \delta), \quad z(0)=z^{\prime}(0)=0, \quad z(\delta) \geq \gamma .
$$

An elementary calculation shows that this boundary value problem has a solution if and only if

$$
c \geq \frac{1}{\sqrt{2 \delta}} \int_{0}^{\gamma} \frac{d z}{\sqrt{G(z)}}=: c^{\prime} .
$$

If $c \geq c^{\prime}$, for $x \in \bar{B}, z(|x|)$ is such that $z(|x|) \geq \gamma$ for $x \in \partial B, \Delta z(|x|)=0$ for $x=$ 0 and, for $|x| \neq 0$

$$
\Delta z=z^{\prime \prime}+\frac{N-1}{|x|} z^{\prime}=c^{2} g(z)+\frac{N-1}{|x|} \int_{0}^{|x|} c^{2} g(z(s)) d s \leq N c^{2} g(z)
$$

where the last inequality follows by (A-2) since $z$ is increasing. Consequently, $z(|x|), x \in \bar{B}$, is an upper solution for (2.18) with $\bar{c}^{2}$ replaced by $N c^{2}$. The method of upper and lower solutions together with the uniqueness of $\bar{u}$ implies that $0 \leq \bar{u} \leq z$, hence we have $\bar{u}(0) \leq z(0)=0$. The assertion is thus established.

PROOF OF THEOREM 2.5. Let $C_{1}, C_{2}$ be two bounded closed disjoint sets related to the admissibility property of $I$. Then $\varepsilon:=\operatorname{dist}\left(C_{1}, C_{2}\right)>0$. Taking $\delta:=\varepsilon / 4$, the set $N:=\left\{x \in D^{-}: \operatorname{dist}\left(x, C_{1} \cup C_{2}\right) \geq \delta\right\}$ is nonempty and the sets $D_{1}:=D \cap\left(C_{1}\right)_{\delta}, D_{2}:=D \cap\left(C_{2}\right)_{\delta}$ are such that $D_{1} \cup D_{2} \cup N=D$ and $D_{1}, D_{2}$, $N$ are disjoint (here for any $A \subseteq \mathbf{R}^{N}, A_{\delta}:=\left\{x \in \mathbf{R}^{N}: \operatorname{dist}(x, A)<\delta\right\}$ ).

Let $U$ be the maximal solution of $\left(\mathrm{P}_{s}\right)_{\lambda}$. We shall prove the existence of $\lambda_{0}>0$, such that for all $\lambda \geq \lambda_{0} U(x)=0$ for all $x \in N$. Thus the function

$$
U^{\prime}:= \begin{cases}U & \text { in } D_{1} \\ 0 & \text { in } N \cup D_{2},\end{cases}
$$

is still a solution of $\left(\mathrm{P}_{s}\right)_{\lambda}$ and belongs to $N_{I}(\lambda)$, i.e. $N_{I}(\lambda) \neq \varnothing$. Indeed by Corollary 2.2(i), there exists $C \in R$, independent of $\lambda$, such that $U \leq C$. For any $x_{0} \in N$, consider a ball $B\left(x_{0}\right)$ of radius $\delta / 2$ centered at $x_{0}$. By definition $U$ is the unique solution to the problem

$$
\begin{cases}\Delta u-\lambda a^{-}(x) g(u)=0 & \text { in } B\left(x_{0}\right) \cap D, \\ u=U & \text { on } \partial\left(B\left(x_{0}\right) \cap D\right) .\end{cases}
$$

Due to the admissibility of $I$ and the definition of $N$, we have $a(x)<0$ for any $x \in \bar{N}_{\delta / 2} \cap \bar{D}$. Hence the continuity of $a$ implies that $\underline{a}>0$ exists, such that 
$\underline{a} \leq a^{-}(x)$ for $x \in \bar{N}_{\delta / 2} \cap \bar{D}$. Let $\bar{u}$ be the solution of $\Delta u=\lambda \underline{a} g(u)$ in $B(0), u=C$ on $\partial B(0)$. In view of $(\mathrm{A}-4)^{\prime}$, Lemma 2.5 applies to prove that $\lambda_{0}>0$ exists, such that $\bar{u}(0)=0$ for any $\lambda \geq \lambda_{0}$. Moreover, for any $x_{0} \in N, \tilde{u}(x):=\bar{u}\left(x-x_{0}\right)(x \in$ $\left.\overline{B\left(x_{0}\right) \cap D}\right)$, is an upper solution for $\left(\mathrm{P}^{-}\right)$. Then a comparison argument yields $0 \leq U\left(x_{0}\right) \leq \tilde{u}\left(x_{0}\right)=\bar{u}(0)=0$ for any $x_{0} \in N$, provided that $\lambda \geq \lambda_{0}$ (see [DH] for details).

COROllary 2.3. Assume (A-1)-(A-3), (A-4)', (A-5)-(A-6). Suppose $\{x \in$ $D: a(x)=0\} \subseteq \partial D^{+}$and $\operatorname{dist}\left(D_{k}^{+}, D_{l}^{+}\right)>\varepsilon>0$ for all $k \neq l$. Let $h$ be the number of connected components of $\bar{D}^{+}$which do not intersect $\overline{\Gamma^{+}}$.

(i) Then for $\lambda \geq \lambda_{0},\left(\mathrm{P}_{s}\right)_{\lambda}$ has exactly $2^{h}$ solutions.

(ii) There exists a number $\lambda_{1}$ such that for all $\lambda \geq \lambda_{1}$ and for all solutions $u$ of $\left(\mathrm{P}_{s}\right)_{\lambda}$ we have $u \equiv U$ in $\operatorname{supp} u, U$ being the maximal solution of $\left(\mathrm{P}_{s}\right)_{\lambda}$.

Remark that in Theorem $2.5 r=+\infty$ is allowed, while the hypotheses in Corollary 2.3 imply $r<+\infty$.

\section{Parabolic case.}

3.1. Regions of attractivity. Consider problem (P) of $\S 1$. The results of this section are based on [dMST] and extend some ideas of [PT, ACP] to problem (P). The aim is to study the asymptotic behavior of the solution of $(\mathrm{P})$. For this purpose we shall need the following

DEFINITION 3.1. An interval $\left[w_{1}, w_{2}\right]:=\left\{w \in L_{+}^{\infty}(D): w_{1} \leq w \leq w_{2}\right\}$ is called $L^{p}$-attractive if there is a set $Q \subseteq L_{+}^{\infty}(D)$ such that:

(i) $\left[w_{1}, w_{2}\right] \subseteq Q$;

(ii) for any $w_{0} \in Q$, the solution $w\left(\cdot, t ; w_{0}\right)$, of problem (P) exists and satisfies $\operatorname{dist}\left\{w\left(\cdot, t ; w_{0}\right),\left[w_{1}, w_{2}\right]\right\} \rightarrow 0$ for $t \rightarrow \infty$ in $L^{p}(D)$.

In the sequel we shall put $w_{I}:=\Phi^{-1}\left(u_{I}\right)$ and $W:=\Phi^{-1}\left(U_{I}\right)=\Phi^{-1}(U)$, where $u_{I}$ and $U$ are the minimal and maximal solutions of $\left(\mathrm{P}_{s}\right)$ in $\mathbf{S}_{I}$. Since $\Phi$ is monotonic we have

$$
w_{I} \leq w \leq W \quad \text { in } D
$$

for any stationary solution $w$ of $(\mathrm{P})$ which is positive in $D_{I}^{+}$. Let us denote by $C_{k}(k=1,2, \ldots, h)$ the connected components of the set $\{x \in \bar{D}: W>0\}$ which do not intersect $\Gamma^{+}$.

THEOREM 3.1. Assume (A-1), (A-6) and (A-7).

(i) $\left[w_{I}, W\right]$ attracts all solutions $w\left(\cdot, t ; w_{0}\right)$ of $(\mathrm{P})$ with $w_{0} \not \equiv 0$ in $C_{k} \cap\left(\bigcup_{i \in I} D_{i}^{+}\right)$ for any $k=1,2, \ldots, h$, in the $L^{p}$-sense $(p$ being any number in $[1, \infty)$ if $N \geq 2$ and $p=\infty$ if $N=1$, or if $w\left(x, t ; w_{0}\right)$ is uniformly continuous in $[\varepsilon, \infty) \times D$ for some $\varepsilon>0)$.

(ii) If $w_{0} \leq w_{I}$ and $w_{0} \not \equiv 0$ in $C_{k} \cap\left(\bigcup_{i \in I} D^{+}\right)$for any $k=1,2, \ldots, h$ then $w\left(\left(\cdot, t ; w_{0}\right) \rightarrow w_{I}\right.$ in $L^{p}(D)$ as $t \rightarrow \infty$ (here $p$ is as in $(\mathrm{i})$ ).

PROOF. Follows from a suitable application of Lemmas 2.3 and 1.1 (see also Theorem 8 in $[\mathbf{P T}])$.

COROLLARY 3.1. Under the assumptions (A-1)-(A-6), if $r<\infty$, for any $w_{0} \not \equiv$ 0 in $D_{i}^{+}(i \in \mathbf{M})$ we have $\lim _{t \rightarrow \infty} w\left(\cdot, t ; w_{0}\right)=W$, where $W$ is the unique stationary solution of $(\mathrm{P})$ which is positive in $D^{+}$. 
PROOF. The proof is a straightforward consequence of Theorems 2.1 and 3.1. In connection with the above corollary, it is worth observing that $W$ may be regarded as "the minimum of the energy" (see Theorem 2.3(ii)).

3.2. Global behavior of the solutions of $(\mathrm{P})$. Throughout this section we shall need the continuity of $w\left(x, t ; w_{0}\right)$ in $Q_{\infty}$. As it was shown by Sacks [S] and Di Benedetto [DB], this is true under very general hypotheses. Under this assumption we have

THEOREM 3.2. Assume (A-1)-(A-6) and suppose that $\mathbf{M}$ is finite. Then for any initial condition $w_{0}, w\left(\cdot, t ; w_{0}\right)$ converges to a stationary solution as $t \rightarrow \infty$.

PROOF. Let us first suppose that for each $k \in \mathbf{M}$ there exists $x_{k} \in D_{k}^{+}$and $t_{k} \geq$ 0 such that $w\left(x_{k}, t_{k} ; w_{0}\right)>0$. Due to the continuity of $w$, Lemmas 2.3 and $1.1(\mathrm{i})$ give $w\left(x_{k}, t ; w_{0}\right)>0$ for all $t \geq \max \left\{t_{1}, \ldots, t_{r}\right\}=: t_{0}$. Hence Corollary 3.1 applies to the function $w\left(\cdot, t+t_{0} ; w_{0}\right)=w\left(\cdot, t ; w\left(\cdot, t_{0} ; w_{0}\right)\right)$ and yields $\lim _{t \rightarrow \infty} w\left(\cdot, t ; w_{0}\right)=$ $W$.

Next we consider the case where $w\left(\cdot, t ; w_{0}\right) \equiv 0$ in $D_{j}^{+}$for any $j=1, \ldots, q$ $(q \leq r)$, for any $t \geq 0$. Then $w$ is a solution of $(\mathrm{P})$ with $a$ replaced by

$$
\tilde{a}:= \begin{cases}0 & \text { in } D_{j}^{+}(j=1, \ldots, q), \\ a & \text { elsewhere }\end{cases}
$$

this problem will be denoted by $(\tilde{\mathrm{P}})$.

Since $w\left(\cdot, t ; w_{0}\right)$ is a solution of $(\tilde{\mathrm{P}})$, we can argue as in the first part of the proof. Hence $w\left(\cdot, t ; w_{0}\right)$ converges to the maximal solution $\tilde{W}$ of $(\tilde{\mathrm{P}})$ as $t \rightarrow \infty$. Since $w(\cdot, t ; w)$ vanishes identically in $D_{1}^{+} \cup \cdots \cup D_{q}^{+}$for any $t \geq 0$, we have $\tilde{W} \equiv 0$ in $D_{1}^{+} \cup \cdots \cup D_{q}^{+}$. Hence $\tilde{W}$ is a stationary solution of $(\mathrm{P})$, too; in fact, $\tilde{W}$ is the unique solution of $\mathbf{N}_{\{q+1, \ldots, r\}}$.

COROLlaRY 3.2. The statement of Theorem 3.2 remains valid if $\mathbf{M}=\mathbf{N}$, provided that for any $I \subseteq \mathbf{M}, \bar{D}_{I}^{+} \subseteq\left(\bigcup_{k \in I} \bar{D}_{k}^{+}\right) \cup \partial D$.

PROOF. We may assume that for any $k \in \mathbf{M}=\mathbf{N}$ there exists $x_{k} \in D_{k}^{+}, t_{k} \geq 0$, such that $w\left(x_{k}, t_{k} ; w_{0}\right)>0$. Otherwise this is true if we replace $(\mathrm{P})$ by $(\tilde{\mathrm{P}})$ as in the proof of Theorem 3.2 , possibly taking $q=+\infty$; the case where $\tilde{\mathbf{M}}$ associated to $(\tilde{\mathrm{P}})$ is finite follows as in Theorem 3.2. Let us consider the sequence $\left\{\underline{w}_{n}\right\}_{n=1}^{\infty}$ where $\underline{w}_{1}:=\underline{w}_{\{1\}}, \ldots, \underline{w}_{n}:=\underline{w}_{\{1, \ldots, n\}}, \ldots$ are the minimal solutions (in $S_{\{1\}}, \ldots, S_{\{1, \ldots, n\}}, \ldots$, respectively) whose existence has been proved in Theorem 2.2 .

Then $\left\{\underline{w}_{n}\right\}_{n=1}^{\infty}$ is nondecreasing and bounded from above by the maximal solution $W$. Hence there exists the pointwise limit $\bar{w} \leq W$ of $\left\{\underline{w}_{n}\right\}_{n=1}^{\infty}$ and an argument similar to that used in the proof of Theorem 2.4, implies that $\underline{w}_{n} \uparrow \bar{w}$ uniformly and that $\bar{w}$ is a stationary solution of $(\mathrm{P})$. Moreover $\bar{w}>0$ in $\bar{D}_{k}^{+}$for any $k \in \mathbf{N}$, hence $\bar{w}=W$ by the uniqueness assumption. Hence $\underline{w}_{n} \uparrow W$ and from Theorem $3.1 w\left(\cdot, t ; w_{0}\right) \rightarrow\left[\underline{w}_{-n}, W\right]$ as $t \rightarrow \infty$, for any $n \in \mathbf{N}$. Hence the proof is complete.

ACKNOWLEDGMENTS. Part of this work was done while one of the authors (C. B.) was visiting the Second University of Rome. She would like to express her gratitude for the hospitality and the stimulating atmosphere. 


\section{REFERENCES}

[ACP] D. G. Aronson, M. Crandall and L. A. Peletier, Stabilization of solutions of a degenerate diffusion problem, Nonlinear Anal. 6 (1982), 1001-1022.

[DB] E. Di Benedetto, Continuity of weak solutions to a general porous medium equation, Indiana Univ. Math. J. 32 (1983), 83-118.

[DH] J. I. Diaz and J. Hernàndez, On the existence of a free bcundary for a class of reaction-diffusion systems, SIAM J. Math. Anal. 15 (1984), 670-685.

[L] T. Laetsch, Uniqueness for sublinear boundary value problems, J. Differential Equations 13 (1973), 13-23.

[GM] M. E. Gurtin and R. C. MacCamy, On the diffusion of biological populations, Math. Biosci. 33 (1977), 35-49.

[dMST] P. De Mottoni, A. Schiaffino and A. Tesei, Attractivity properties of nonnegative solutions for a class of nonlinear degenerate parabolic problems, Ann. Mat. Pura Appl. 136 (1984), 35-48.

$[\mathrm{PeT}]$ L. A. Peletier and A. Tesei, Global bifurcation and attractivity of stationary solutions of a degenerate diffusion equation, Adv. in Appl. Math. 7 (1986), 435-454.

[PT] M. A. Pozio and A. Tesei, Support properties of solution for a class of degenerate parabolic problems, Comm. Partial Differential Equations 12 (1987), 47-75.

[N] T. Namba, Density-dependent dispersal and spatial distribution of a population, J. Theor. Biol. 86 (1980), 351-363.

[S] P. Sacks, The initial and boundary value problem for a class of degenerate parabolic equations, Comm. Partial Differential Equations 8 (1983), 693-733.

[Sch] M. Schatzman, Stationary solutions and asymptotic behaviour of a quasilinear degenerate parabolic equation, Indiana Univ. Math. J. 33 (1984), 1-30.

[Sp] J. Spruck, Uniqueness in a diffusion model of population biology, Comm. Partial Differential Equations 8 (1983), 1605-1620.

MATHEMATISCHES INSTITUT, UNIVERSITÄT BASEL, RHEINSPRUNG 21, CH-4051 BASEL, SWITZERLAND

Dipartimento di MATEMATICA, II UniVersità di ROMA, Via O. RAIMONDO, I-00173 ROMA, ITALY 